\title{
Higher-order statistics of a bistable system driven by dichotomous colored noise
}

\author{
Tilo Schwalger \\ Humboldt-Universität Berlin, Newtonstrasse 15, 12489 Berlin, Germany \\ and RIKEN Brain Science Institute, Hirosawa 2-1, Wako, Saitama 351-0198, Japan \\ Benjamin Lindner \\ Max-Planck-Institut für Physik Komplexer Systeme, Nöthnitzer Strasse 38, 01187 Dresden, Germany \\ (Received 6 March 2008; revised manuscript received 11 June 2008; published 18 August 2008)
}

\begin{abstract}
Exact expressions for first- and higher-order residence time statistics, count statistics, and spectral properties of a bistable system driven by dichotomous colored noise are put forward and discussed. The employed method is based on a discrete kinetic scheme and is valid for a wide range of parameter values of the colored noise. This permits a detailed analysis of the effects of noise correlations for arbitrary correlation times. It is found that at a characteristic correlation time of the dichotomous noise, the residence time sequence becomes Poissonian; in particular, all correlations between residence times disappear. We also find that correlations become strongest for a finite strength of the driving force. The analytical results can also be used to infer the underlying driving parameters in the case of noise with long-range temporal correlations.
\end{abstract}

DOI: 10.1103/PhysRevE.78.021121

PACS number(s): 05.40.-a, 05.10.Gg

\section{INTRODUCTION}

The noise-assisted escape from a metastable state is one of the basic problems in statistical physics. The problem is encountered in different fields, such as, for instance, chemical physics (reaction rate problem), biological physics (conformational changes of proteins), and neurobiology (stochastic firing of action potentials by a nerve cell). There exists a mathematical apparatus (Langevin equation, Fokker-Planck, and master equations) by which escape processes under different conditions (see below) can be faithfully described and analyzed.

As a matter of fact, many escape processes repeat themselves over and over again. This is true, for instance, for the escape from the resting state in excitable systems such as neurons-here the escape is associated with the occurrence of a spike (neural action potential) after which the dynamics is brought back via a recovery state to the resting state from which it started. Another example is the Brownian motion in a periodic potential (a model for an impressive number of different physical systems, see [1]). Here the metastable states are the (infinitely many) potential minima. Last but not least, a bistable system as given by the potential in Fig. 1(a) provides one of the simplest example in which we can have a sequence of escape events, namely the noise-driven transitions from the left to the right and from the right to the left minima.

In all the above examples, the statistics of the escape process is completely characterized by the sequence of residence times $(R T)$ (together with the information in which state the RT was spent if this is ambiguous). Another statistics with the same information (although not intuitive in every system) is the count statistics for the number of transitions within a certain time window. Most of the aforementioned work has focused solely on the statistics of the mean number of escapes (if divided by the time window, this gives the escape rate) or the statistics of a single RT (the mean of which is equal to the inverse escape rate). Higher-order statistics, however, can reveal important aspects of the underlying dy- namics. This has been recognized in neuroscience, where intervals between adjacent action potentials (corresponding to first-passage times or residence times) can exhibit strong correlations [2-4]. Theoretical studies have characterized these correlations [5-7] and have elucidated their biological function for an enhanced signal transmission [3,8-10]. Outside the neural context, however, correlations among residence times are still largely unexplored.

Let us recall the classical Kramers problem for the escape in a symmetric bistable potential as sketched in Fig. 1(a) to illustrate why researchers were not interested in higher-order
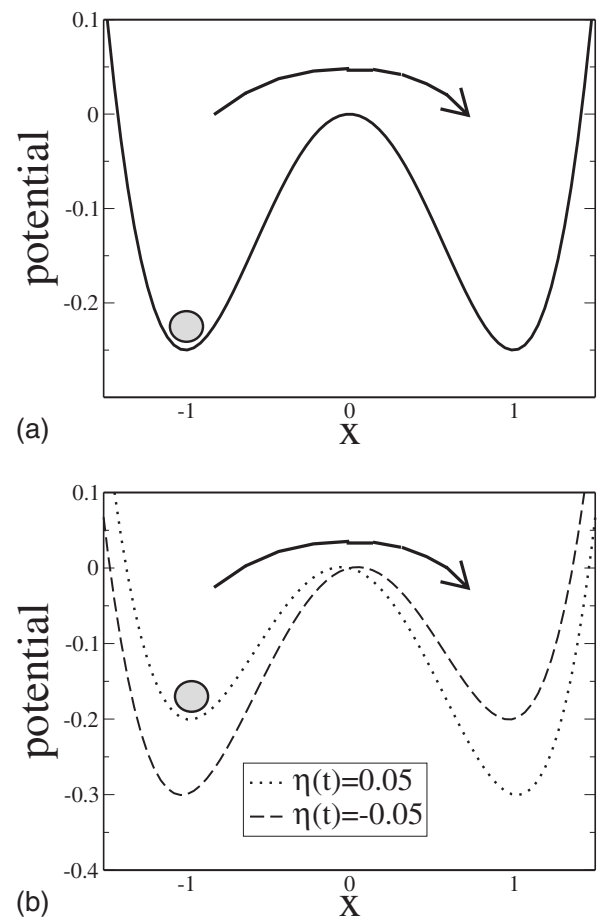

FIG. 1. Bistable dynamics with a noise-induced escape over the potential barrier at $x=0$. Unperturbed dynamics in (a) and the bistable dynamics with dichotomous fluctuations in (b). 
statistics. Driven only by thermal fluctuations, the escape rate for this system has been studied by Kramers [11] and others (see [12]). The simpler overdamped case is described by only one Langevin equation,

$$
\dot{x}=-U^{\prime}(x)+\sqrt{2 D} \xi(t) .
$$

Here we have set the friction constant (the prefactor of the time derivative) to 1 ; inertia (the second temporal derivative) has been neglected. The potential $U(x)$ should be symmetric around zero and the thermal fluctuations with noise intensity $D$ are uncorrelated $\left\langle\xi(t) \xi\left(t^{\prime}\right)\right\rangle=\delta\left(t-t^{\prime}\right)$. Kramers and others focused on the problem to calculate the rate of escape out of one of the minima across the barrier [the potential maximum at $x=0$ in Fig. 1(a)] in the limit of weak fluctuations $(D$ $\ll \Delta U$ ) and came up with the famous result

$$
r=\frac{\sqrt{U^{\prime \prime}\left(x_{\min }\right)\left|U^{\prime \prime}\left(x_{\max }\right)\right|}}{2 \pi} \exp [-\Delta U / D] .
$$

For weak noise, the rate $r$ is indeed all we have to know. First of all, the limit of weak noise implies that the residence time distribution follows a rare-time statistics, i.e., an exponential distribution that is completely determined by just one parameter-the rate $r$. Secondly, it follows from the Markov character of the system Eq. (1) that the residence times are uncorrelated-once the particle has crossed the barrier, it does not have any memory about this past escape event and about the time (residence time) spent in the previous minimum. Hence the length of one residence time cannot affect that of the following intervals and all the residence times in the sequence are mutually independent. The sequence of escape events forms a renewal point process that is moreover (because of the exponential residence time density) a Poisson process for which also the counting statistics is straightforward. Hence, in the case of a purely white-noise driven escape the rate is the only parameter we have to know. Higherorder correlations in the RT sequence are simply not present in the overdamped variant of the classical Kramers problem. We note that for the moderately damped and underdamped problem, we expect that the RT sequence displays correlations, however in this case already the calculation of the escape rate is sufficiently difficult.

It should be mentioned that a trivial kind of correlation is introduced in the overdamped case in an asymmetric potential [e.g. one of the lines in Fig. 1(b)]-here a shorter interval (escape over the smaller barrier) is usually followed by a longer interval (escape over the higher barrier). Put differently, in an asymmetric system the particle keeps a memory of the side it is on or was on a certain number of transitions ago. We note that in this case taking only the subsequent residence times in one specific (e.g., the left) well would yield a sequence of uncorrelated times. Another statistics that excludes this trivial kind of correlation is the sequence of stochastic periods, which are simply the sum of two subsequent residence times in the left and right state. The stochastic periods (also indicated in Fig. 2) are a natural generalization of what would be the period for a deterministic two-state process.

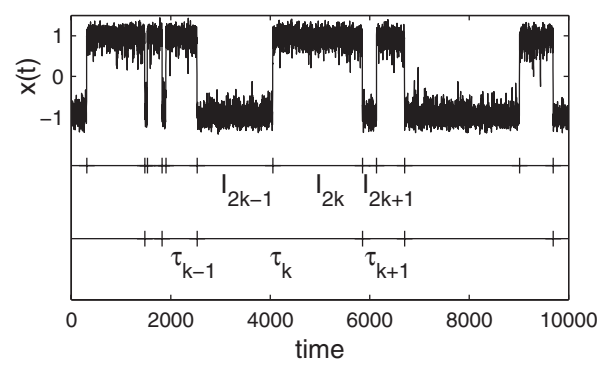

FIG. 2. Illustration of residence times and stochastic periods. From top to bottom: (i) typical trajectory of the driven bistable system $\left(D=0.05, \sigma=0.04, \lambda=0.1 \gamma=1.329 \times 10^{-4}\right)$; (ii) associated sequence of RT's $I_{n}$; (iii) sequence of stochastic periods $\tau_{n}$.

Extensions of the model Eq. (1) have been considered in the context of stochastic resonance $[13,14]$, resonant activation [15], and other noise-induced resonances (e.g., in systems with delay as in Refs. [16,17]). Generally, a complex system like a protein which performs an escape out of a metastable state may have other processes of internal (feedback) or external (a signal to be transduced in a biological system) origin that perturb this process. Also fluctuations may not be white as assumed in Eq. (1). All these cases have been studied with particular emphasis on changes in the escape rate or in the distribution of residence times. Almost no attention has been paid to the fact that generally, with a colored noise input, a delayed feedback, or an external (nonwhite) perturbation, the conditions for the independence of the RT's in the sequence are violated. The properties of the RT correlations may characterize aspects of the escape process much better than the rate of escape (equivalently, the mean residence time). However, to calculate these correlations is much more complicated than the single RT statistics, which is most likely the reason that there have been only a few such attempts.

We have recently shown [18] how for a bistable system with dichotomous noise perturbation [for the modulated potential, see Fig. 1(b)] the statistics of the correlation can be calculated. The approach used a discretization and particular master equation setup that allows for an analytical treatment. We also showed that RT correlations oscillate and decay in magnitude exponentially with increasing lag. Furthermore, under a resonance-like condition (the switching rate of the dichotomous noise matches the escape rate of the unperturbed system) these correlations vanish at all lags and reappear for fast driving with an inverted amplitude (e.g., lags that are positively correlated for slow driving become negatively correlated for fast driving). We have also demonstrated how one could infer a possibly unknown switching rate from the correlation statistics in the case of a slow driving force.

Here we detail our approach and discuss also other measures of the driven process, such as, for instance, the correlations of the stochastic periods, the Fano factor of the number of transitions, and the power spectrum of the bistable process. We also present proof of the formula for the SCC, the main result of [18]. Our results are exact within the framework of a rate description and apply for arbitrary correlation times; they are moreover not limited to a weak driving force. Our approach only assumes the use of dichoto- 
mous colored noise, which can be seen as a case study to explore the whole range of correlation times, including longrange temporal correlations. We hope that the general results derived in this study will help to clarify the influence of temporal correlations for first- and higher-order statistics.

\section{MODEL AND NTH-ORDER RT STATISTICS}

\section{A. Stochastically driven bistable system}

Let us consider the standard example of an overdamped Brownian motion $x(t)$ in the doublewell potential $U(x)$ $=x^{4} / 4-x^{2} / 2$. Additionally, we introduce a stochastic driving force $\eta(t)$, so that Eq. (1) reads

$$
\dot{x}=-U^{\prime}(x)+\eta(t)+\sqrt{2 D} \xi(t) .
$$

Here, $\eta(t)$ is assumed to be a dichotomous Markov process with exponential correlation function $\left\langle\eta(t) \eta\left(t^{\prime}\right)\right\rangle$ $=\sigma^{2} \exp \left(-2 \lambda\left|t-t^{\prime}\right|\right)$. This means that $\eta(t)$ switches between the two values $\sigma$ and $-\sigma$ with a constant rate $\lambda$, which leads effectively to a time-dependent, asymmetric potential $U$ $\rightarrow U \mp \sigma x$ as shown in Fig. 1(b). The amplitude $\sigma$ of the dichotomous driving is such that the system preserves bistability. Specifically, this requires $\sigma<2 /(3 \sqrt{3})$.

The white Gaussian noise $\xi(t)$ permits stochastic transitions between the two metastable states. In the symmetric case, i.e., when $\sigma=0$, they are separated by a potential barrier of height $\Delta U=1 / 4$ and the minima of the potential are located at $x=-1$ and 1 (corresponding to "left" and "right" metastable state, respectively). In the presence of the dichotomous driving, the barrier heights for a transition are smaller or larger than $\Delta U=1 / 4$ depending on the metastable state (left or right) and the current value of $\eta(t)$. Note that the locations of the minima also change compared to the symmetric case.

Due to the stochastic nature of the driving force, $\eta(t)$ can be seen as a simple realization of colored noise with a correlation time $1 /(2 \lambda)$; see, e.g., [19]. The advantage of this combination of white and two-state noise consists in analytically feasible calculations that permit studying the effect of noise correlations. In a different context, similar setups have successfully served as a model for stochastic synchronization with periodic [20] or stochastic [21] driving or both periodic and stochastic driving [22]. It should be also mentioned that Eq. (3) is a simple model of one bistable system (modeled by the dichotomous input) driving another one. There exist also possible experimental realizations $[23,24]$.

To judge the validity of our analytical results, we performed extensive numerical simulations of the continuous dynamics $x(t)$, from which we obtained a long sequence of RT's (the number of RT's in one sequence was at least $10^{7}$ ). The white noise driven dynamics in the potential $U(x)$ $-\eta(t) x$ was integrated using the Heun algorithm [25] with step size $\Delta t=0.01$. The dichotomous switchings of the potential were realized by the Gillespie algorithm [26], i.e., the waiting times were randomly drawn from an exponential distribution. In Fig. 2, we show a typical trajectory of the bistable system (3), which jumps between the two metastable states. It becomes apparent that many physical features, such

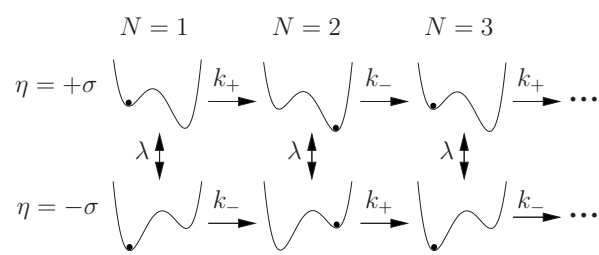

FIG. 3. Kinetic scheme of the discrete state process. The dichotomous driving is described by the two states $\eta= \pm \sigma$, which change with rate $\lambda$. The bistable system can perform a transition to the other metastable state with rate $k_{+}$or $k_{-}$depending on the driving state $\eta(t)$, thereby incrementing the state variable $N(t)$ by one. Adopted and modified from [18].

as the RT's, can still be captured if one neglects the fast intrawell dynamics. This permits us to formulate the problem in the framework of a rate model with two discrete states $X_{-}$ and $X_{+}$corresponding to left and right metastable state, respectively. More precisely, if the trajectory belongs to the basin of attraction of the left attractor of the bistable potential, the system is said to be in the state $X_{-}$, otherwise it is said to be in the state $X_{+}$. There is an exception, however: in order to enter a new state, the trajectory must first reach the attractor (i.e., the minimum of the potential) belonging to this new state. Thus, a short fluctuation into the other basin of attraction without reaching the attractor and back to the original domain does not count as a state transition. This definition of a transition is used to define the RT's, which are simply the time intervals between two successive transitions. Hence, a sequence of RT's is essentially a sequence of first passage times from one attractor to the other. In the same manner, we also extracted the RT sequence from the simulations of $x(t)$.

The two states $X_{+}$and $X_{-}$of the process $x(t)$ together with the two states $+\sigma$ and $-\sigma$ of the dichotomous noise $\eta(t)$ form a four-state stochastic process. However, instead of using the variable $x(t)$, we introduce the counting variable $N(t)$. This variable labels successively the metastable states by an integer $n$ starting with $N(0)=1$ corresponding to the state $X_{-}$. Here, we assumed without loss of generality that the system starts in the left metastable state $X_{-}$. Each transition increments $N(t)$ by one. Consequently, an odd value of $N(t)$ is equivalent to $X_{-}$, whereas an even value of $N(t)$ is equivalent to $X_{+}$. The usage of $N(t)$ instead of $x(t)$ will allow us to count the number of transitions.

As a next step, we specify the statistics of the transition times between the discrete states. According to the definition of a transition, the statistics are, in general, given by firstpassage-time densities from one attractor to the other. After a short relaxation period following a transition, the process has established a local equilibrium near the attractor from which the system escapes with an exponentially distributed waiting time. If the mean RT of each metastable state is much larger than the local relaxation time $t_{\mathrm{loc}} \sim\left[U^{\prime \prime}\left(x_{\min }\right)\right]^{-1}$, we can neglect the transient period. This allows us to use a rate description with constant transition rates equal to the escape rates of the exponential waiting time density. As illustrated in Fig. 3, the transition probabilities between states per unit time are denoted by $k_{ \pm}=W(n, \pm \sigma \mid n-1, \pm \sigma)$ if $n$ is even, $k_{\mp}=W(n, \pm \sigma \mid n-1, \pm \sigma)$ if $n$ is odd, and 
$\lambda=W(n, \pm \sigma \mid n, \mp \sigma)$. Note that these rates contain all the details of the underlying continuous model (e.g., shape of the potential, amplitude of the dichotomous driving, locations of the minima of the potential).

The probabilistic dynamics of the bistable system is completely characterized by the transition rates and the initial conditions of the system. In particular, the dynamics can be formulated by a master equation: If $p_{n, \pm}(t)$ denotes the joint probability that at time $t$ the system is in the $n$th metastable state, i.e., $N(t)=n$, and $\eta(t)= \pm \sigma$, the master equation reads for odd $n$ (cf. Fig. 3)

$$
\begin{aligned}
& \dot{p}_{n,+}=-\left(k_{+}+\lambda\right) p_{n,+}+k_{-} p_{n-1,+}+\lambda p_{n,-}, \\
& \dot{p}_{n,-}=-\left(k_{-}+\lambda\right) p_{n,-}+k_{+} p_{n-1,-}+\lambda p_{n,+} .
\end{aligned}
$$

Here and in the following, the equations for even $n$ are obtained by exchanging the roles of $k_{+}$and $k_{-}$.

The determination of the initial conditions $p_{n, \pm}(0)$ needs some more explanation. What complicates matters is that our master equation approach requires an ensemble for the initial conditions such that the ensemble average coincides with the average over the sequence of RT's. In other words, the initial conditions of the ensemble should correspond to the state of the system at transitions along a single trajectory of the system. This can be achieved by regarding a subensemble of the stationary system whose members have all just suffered a transition from $X_{+}$to $X_{-}$at the initial moment $t=0$. In doing so, we find, for instance, that the ensemble average of the transition time of the following transition (from $X_{-}$to $X_{+}$) gives exactly the mean RT of a single RT sequence. The particular preparation of the ensemble implies that the probabilities to find the initial driving force $\eta(0)$ at $+\sigma$ or $-\sigma$ are not equal, because the initial transition from $X_{+}$to $X_{-}$is more likely to occur when the potential is tilted to the left, i.e., when $\eta(0)=-\sigma$. Thus, the initial probability $p_{1, \pm}(0)$ is given by the probability that $\eta(0)= \pm \sigma$, which is exactly the probability of $\eta(t)$ sampled at the transition times $t=t_{n}$ (biased sampling). Furthermore, the initial probabilities for $n>1$ are zero, i.e., $p_{n, \pm}(0)=0$, since at time $t=0$ the system is in the first metastable state $N(0)=1$.

The probability that $\eta=\sigma$ at the moment of the initial transition is proportional to the probability current $j(\sigma)$ from the state $\left\{X_{+}, \sigma\right\}$ into the state $\left\{X_{-}, \sigma\right\}$, i.e., $p_{1,+}(0)=c j(\sigma)$ ( $c$ is a factor of proportionality). Analogously, the other initial probability is $p_{1,-}(0)=c j(-\sigma)$. To determine the currents $j(\sigma)$ and $j(-\sigma)$, we consider the stationary probabilities $p_{s}\left(X_{+}, \sigma\right)=\Sigma_{m} p_{2 m,+}(t)$ and $p_{s}\left(X_{+},-\sigma\right)=\Sigma_{m} p_{2 m,-}(t)$ to find the system in the lower right or upper right well of the bistable potential. To this end, we sum up both sides of the master equation (4) over all even integers $n$ and use $p_{s}\left(X_{-}, \pm \sigma\right)$ $=p_{s}\left(X_{+}, \mp \sigma\right)$ to obtain an equation for the stationary probabilities,

$$
\left(k_{+}+\lambda\right) p_{s}\left(X_{+},-\sigma\right)=\left(k_{-}+\lambda\right) p_{s}\left(X_{+}, \sigma\right) .
$$

With the additional relation $p_{s}\left(X_{+}, \sigma\right)+p_{s}\left(X_{+},-\sigma\right)=\frac{1}{2}$, we find

$$
p_{s}\left(X_{+}, \pm \sigma\right)=\frac{\lambda+k_{ \pm}}{2\left(k_{+}+k_{-}+2 \lambda\right)} .
$$

The probability currents are then simply given by $j(\sigma)$ $=k_{-} p_{s}\left(X_{+}, \sigma\right)$ and $j(-\sigma)=k_{+} p_{s}\left(X_{+},-\sigma\right)$. Using the normalization relation $p_{1,+}(0)+p_{1,-}(0)=1$, one can eliminate the factor $c$, which finally gives the initial probabilities

$$
p_{1, \pm}(0)=\frac{k_{\mp}\left(\lambda+k_{ \pm}\right)}{\lambda\left(k_{+}+k_{-}\right)+2 k_{+} k_{-}} .
$$

As long as a rate description is meaningful, the discrete bistable model (4) describes the continuous model Eq. (3) faithfully if the transition rates $k_{+}$and $k_{-}$are adequately specified as the inverse mean first passage times from one minimum to the other minimum of the double-well potential. They are given by the well-known formula for the mean first passage time (MFPT) of a one dimensional diffusion process [27],

$$
k_{ \pm}=D\left[\int_{a}^{b} d y e^{V_{ \pm}(y) / D} \int_{-\infty}^{y} e^{-V_{ \pm}(z) / D} d z\right]^{-1},
$$

where $V_{ \pm}(x)=U(x) \mp \sigma x$ and $a$ and $b$ are the positions of the left and right minimum of $V_{ \pm}(x)$, respectively, i.e., $a<b$. In the case of small driving amplitude $\sigma$ and small noise intensity $D$, we can approximate the transition rates by the Kramers rates [see Eq. (2)]

$$
k_{ \pm}=\frac{1}{\sqrt{2} \pi} \exp \left(-\frac{\Delta U \mp \sigma}{D}\right) .
$$

\section{B. First- and higher-order RT statistics}

In the following, we define and classify the statistical measures that will be used to characterize the statistics of a RT sequence. To this end, we denote the RT of the $n$th metastable state by $I_{n}$ and the $n$th transition time by $T_{n}$ [the initial (zeroth) transition occurs at $\left.T_{0}=0\right]$. We furthermore define the stochastic periods as

$$
\tau_{n}=I_{2 n-1}+I_{2 n},
$$

i.e., the sum of two subsequent residence times (left and right well), which can be interpreted as one (random) period of the stochastic oscillation (Fig. 2).

In the theory of point processes, the sum of $n$ successive intervals in a sequence is known as the $n$ th-order interval. Due to the special choice of the ensemble, the ensemble average of the transition times $\left\langle T_{n}\right\rangle$ is equal to the $n$ th-order intervals averaged along the sequence. To perform the ensemble averages, we introduce the probability density $f_{n}(t)$ of the $n$th transition time $T_{n}$.

To relate this probability density to the joint probabilities $p_{n, \pm}(t)$, we consider the probability

$$
P_{n}(t)=p_{n,+}(t)+p_{n,-}(t)
$$

that at time $t$ the system is in the $n$th metastable state. Using the master equation (4), we then find that the probability density for odd $n$ is given by 


$$
\begin{aligned}
f_{n}(t) & =-\frac{d}{d t} \operatorname{Prob}\left(t<T_{n}\right) \\
& =-\frac{d}{d t} \sum_{k=1}^{n} P_{k}(t) \\
& =k_{+} p_{n,+}(t)+k_{-} p_{n,-}(t) .
\end{aligned}
$$

We call the RT statistics a first-order RT statistics if it only involves the probability density $f_{1}(t)$, i.e., the statistics of $T_{1}$ (first-order intervals). Conversely, if the RT statistics requires the statistics of $T_{n}$ ( $n$ th-order intervals) with $n>1$, which is given by $f_{n}(t)$, we refer to it as a higher-order $R T$ statistics.

The RT density $f_{1}(t)$ was explicitly calculated and discussed in [18], from which, in general, any first-order RT statistics can be derived; it reads

$$
f_{1}(t)=c_{1} e^{-\alpha_{1} t}+c_{2} e^{-\alpha_{2} t}
$$

with $\quad \alpha_{1 / 2}=k+\lambda \pm \sqrt{k^{2}+\lambda^{2}-\gamma^{2}} \quad$ and $\quad c_{1 / 2}=f_{1}(0) / 2$ $\pm\left[(k+\lambda) f_{1}(0)-\left(2 k \lambda+\gamma^{2}\right)\right] / 2 \sqrt{k^{2}+\lambda^{2}-\gamma^{2}}$. As an important example, we will analyze the coefficient of variation $(\mathrm{CV})$, which constitutes a useful measure of the variability of a point process' intervals. It is defined as the ratio of standard deviation and mean of the RT's,

$$
C_{V}=\frac{\sqrt{\left\langle\Delta T_{1}^{2}\right\rangle}}{\left\langle T_{1}\right\rangle}
$$

Throughout the paper, we use the notation $\left\langle\Delta X^{2}\right\rangle=\left\langle X^{2}\right\rangle$ $-\langle X\rangle^{2}$ for the variance of a random variable $X$. For comparison, the $\mathrm{CV}$ of a Poisson process is 1 and that of a strictly periodic process is zero; a CV larger than 1 indicates strong variability as, for instance, associated with bursting in the number of events.

For a renewal point process, i.e., a point process where all intervals (RT's) are statistically independent, the first-order RT statistics is sufficient, because the process is completely determined by the interval density $f_{1}(t)$. The dichotomous noise in our model, however, induces correlations between RT's, which render the sequence nonrenewal. A higher-order RT measure which quantifies the correlation between two RT's as a function of their lag $n$ is the serial correlation coefficient (SCC) defined by

$$
\rho_{n}=\frac{\left\langle I_{k} I_{k+n}\right\rangle-\left\langle I_{k}\right\rangle\left\langle I_{k+n}\right\rangle}{\left\langle I_{k}^{2}\right\rangle-\left\langle I_{k}\right\rangle^{2}} .
$$

Thus, the $n$th SCC is the covariance between the RT $I_{k}$ and the RT which is lagged by $n, I_{k+n}$, normalized by the variance of $I_{k}$. Here, the involved averages are taken across the sequence (i.e., averaging over the index $k$ ). There is a simple relation, which links the SCC with the $n$ th-order intervals [7],

$$
\rho_{n}=\frac{\left\langle\Delta T_{n+1}^{2}\right\rangle+\left\langle\Delta T_{n-1}^{2}\right\rangle}{2\left\langle\Delta T_{1}^{2}\right\rangle}-\frac{\left\langle\Delta T_{n}^{2}\right\rangle}{\left\langle\Delta T_{1}^{2}\right\rangle} .
$$

Below we will also consider the serial correlation coefficient of the stochastic periods,

$$
\hat{\rho}_{n}=\frac{\left\langle\tau_{k} \tau_{k+n}\right\rangle-\left\langle\tau_{k}\right\rangle\left\langle\tau_{k+n}\right\rangle}{\left\langle\tau_{k}^{2}\right\rangle-\left\langle\tau_{k}\right\rangle^{2}} .
$$

By expressing the periods $\tau_{k}$ by the residence times, one finds the following relation between $\rho_{n}$ and $\hat{\rho}_{n}$ :

$$
\hat{\rho}_{n}=\frac{\rho_{2 n-1}+2 \rho_{2 n}+\rho_{2 n+1}}{2\left(1+\rho_{1}\right)} .
$$

Another possibility to characterize the RT sequence yields the statistics of the count process. This process is given by the number of transitions $N(t)$ that fall in an arbitrarily chosen time window of length $t$. Note that at the beginning of the time window, there does not have to be a transition. A widely used measure yields the Fano factor $F(t)$, which is defined as the variance-to-mean ratio of $N(t)$,

$$
F(t)=\frac{\left\langle\Delta N(t)^{2}\right\rangle}{\langle N(t)\rangle} .
$$

It is a measure for the variability of a point process on a certain time scale $t$ and is equal to unity for a Poisson process. As will become clear below, the Fano factor is a higherorder measure, because it involves the probability densities $f_{n}(t)$ of all $n \geqslant 1$. The calculation of the variance $\left\langle\Delta N(t)^{2}\right\rangle$ also suggests the introduction of an effective diffusion coefficient of the count process,

$$
D_{\text {eff }}=\lim _{t \rightarrow \infty} \frac{d}{d t} \frac{\left\langle\Delta N(t)^{2}\right\rangle}{2} .
$$

This quantity describes the long-time variability of the count process,

$$
F(t) \stackrel{t \rightarrow \infty}{\longrightarrow} 2 D_{\text {eff }}\left\langle T_{1}\right\rangle .
$$

\section{THEORY}

\section{A. Discrete state dynamics}

At first we derive an equation for $f_{n}(t)$ and $P_{n}(t)$ from Eq. (4). For this purpose, we differentiate Eqs. (10) and (11) and apply the master equation (4). Inserting the inverse relation $p_{n, \pm}= \pm\left(f_{n}-k_{\mp} P_{n}\right) /\left(k_{+}-k_{-}\right)(n$ odd $)$, we arrive at

$$
\begin{gathered}
\dot{f}_{n}=-2(\lambda+k) f_{n}+\left(2 \lambda k+\gamma^{2}\right) P_{n}+\gamma^{2} P_{n-1}, \\
\dot{P}_{n}=-f_{n}+f_{n-1},
\end{gathered}
$$

with $f_{0}=P_{0}=0$. Here, we have introduced the parameters $\gamma$ $=\sqrt{k_{+} k_{-}}$and $k=\left(k_{+}+k_{-}\right) / 2$. The meaning of these new parameters becomes particularly clear in the Kramers case given by Eq. (8). In this case, we find

$$
\begin{gathered}
\gamma=\exp (-\Delta U / D) / \sqrt{2} \pi, \\
k=\cosh (\sigma / D) \gamma .
\end{gathered}
$$

Apparently, $\gamma$ coincides with the Kramers rate of the undriven (symmetric) system, i.e., with the escape rate for $\sigma$ $=0$. By contrast, $k$ reflects the driving amplitude $\sigma$ and thus 
TABLE I. Parameter values of the driving amplitude $\sigma$ that were used in the simulations of the Langevin equation (3) with noise intensity $D=0.05$. The table translates $\sigma$ into the parameter $k=\left(k_{-}\right.$ $\left.+k_{+}\right) / 2$ and $\gamma=\sqrt{k_{-} k_{+}}$of the theory. The values of $k$ and $\gamma$ were computed from the MFPT formula (7). For comparison, we also indicate the value of $k_{\mathrm{Kr}} / \gamma_{\mathrm{Kr}}$ obtained by the Kramers rates Eq. (8). Furthermore, the ratio of the rates $k_{+}$and $k_{-}$is shown. For all values of $\sigma$, we obtain $\gamma_{\mathrm{Kr}}=0.00152$. The amplitudes of the dichotomous driving are always smaller than the critical forcing amplitude $\sigma$ $<\sigma_{\text {crit }}=2 /(3 \sqrt{3}) \approx 0.385$, beyond which there is only one potential minimum.

\begin{tabular}{lcccc}
\hline \hline \multicolumn{5}{c}{$D=0.05$} \\
\hline$\sigma$ & $\gamma$ & $k / \gamma$ & $k_{\mathrm{Kr}} / \gamma_{\mathrm{Kr}}$ & $k_{+} / k_{-}$ \\
\hline 0 & 0.001370 & 1.00 & 1.00 & 1.00 \\
0.04 & 0.001329 & 1.30 & 1.34 & 4.54 \\
0.07 & 0.001247 & 2.02 & 2.15 & 14.25 \\
0.1585 & 0.000844 & 10.00 & 11.92 & 398.00 \\
\hline \hline
\end{tabular}

describes the asymmetry of the bistable system caused by the driving force. In general, it holds that $k \geqslant \gamma$, with equality in the symmetric case $\sigma=0$. Thus, the degree of asymmetry induced by the driving amplitude may be quantified by the ratio $k / \gamma \geqslant 1$. Examples for parameter values $k$ and $\gamma$ for the driving amplitudes $\sigma$ that were used in simulations are listed in Table I (cf. also Fig. 4).

The initial conditions for Eq. (20) can be easily derived from Eq. (6) as $f_{1}(0)=\gamma^{2}(\lambda+k) /\left(\lambda k+\gamma^{2}\right), P_{1}(0)=1$, and $f_{n}(0)=P_{n}(0)=0$ for all $n>1$.

At this point, one can see that the two driving parameters $\lambda$ and $k$ enter symmetrically into the dynamics (20) and the initial conditions. This means that exchanging $\lambda$ and $k$ results in exactly the same statistics of the driven bistable system. However, the roles of $k$ and $\lambda$ are not ambiguous in the slow driving case $\lambda<\gamma$, which is due to the definition of $k$ : in contrast to $\lambda$, which can take any non-negative value, $k$ is bounded below by the value $\gamma$. The symmetry entails the interesting consequence that if the switching rate $\lambda$ of the dichotomous driving is equal to $\gamma$, the RT sequence must be a Poisson process with rate $\gamma$. In fact, due to the symmetry, the case $\lambda=\gamma$ for some $k=k^{*}$ is equivalent to $k=\gamma$ and $\lambda$ $=k^{*}$. The situation $k=\gamma$ is, however, precisely the case of the undriven bistable system. By definition, this symmetric system generates a Poisson process with rate $k_{+}=k_{-}=\gamma$.

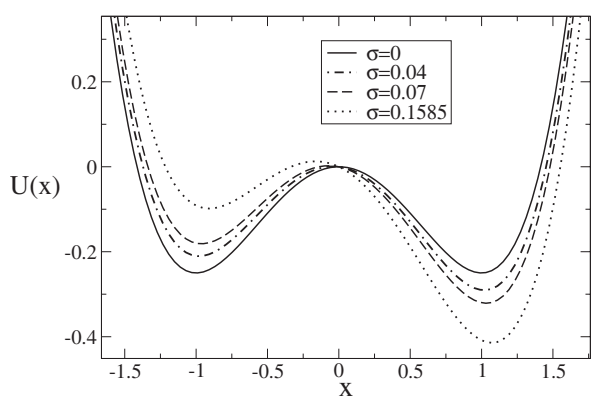

FIG. 4. Asymmetry of the potential for the $\sigma$ values indicated in Table I.
The first and second moment of $T_{n}$, which are needed for the calculation of the CV and SCC, can be obtained from the Laplace transform $\bar{f}_{n}$ defined as

$$
\bar{f}_{n}(s)=\int_{0}^{\infty} f_{n}(t) e^{-s t} d t
$$

As is well known, the moments are given by the derivatives at $s=0$,

$$
\left\langle T_{n}\right\rangle=-\left.\frac{d \bar{f}_{n}}{d s}\right|_{s=0}, \quad\left\langle T_{n}^{2}\right\rangle=\left.\frac{d^{2} \bar{f}_{n}}{d s^{2}}\right|_{s=0} .
$$

We transform the system of differential equations (20) by means of Eq. (21) into the system of linear algebraic equations,

$$
\begin{gathered}
{[s+2(\lambda+k)] \bar{f}_{n}-\left(2 \lambda k+\gamma^{2}\right) \bar{P}_{n}=f_{n}(0)+\gamma^{2} \bar{P}_{n-1},} \\
\bar{f}_{n}+s \bar{P}_{n}=P_{n}(0)+\bar{f}_{n-1},
\end{gathered}
$$

with $\bar{f}_{0}=\bar{P}_{0}=0$.

\section{B. Mean RT and coefficient of variation}

The calculation for the first-order RT statistics can be achieved by solving Eq. (23) for $n=1$, which yields

$$
\begin{aligned}
& \bar{f}_{1}(s)=g(s)\left[\gamma^{2}+2 k \lambda+f_{1}(0) s\right], \\
& \bar{P}_{1}(s)=g(s)\left[s-f_{1}(0)+2(k+\lambda)\right]
\end{aligned}
$$

with $g(s)=\left[s^{2}+2(k+\lambda) s+2 k \lambda+\gamma^{2}\right]^{-1}$. Using Eq. (22), we find the first two moments

$$
\left\langle T_{1}\right\rangle=\frac{k+\lambda}{k \lambda+\gamma^{2}}
$$

and

$$
\left\langle T_{1}^{2}\right\rangle=2 \frac{2\left(k^{2}+\lambda^{2}\right)-\gamma^{2}+3 k \lambda}{\left(k \lambda+\gamma^{2}\right)\left(2 k \lambda+\gamma^{2}\right)} .
$$

The mean transition rate of the driven system is therefore $r$ $=\left(k \lambda+\gamma^{2}\right) /(k+\lambda)$. For slow driving $\lambda<\gamma$ the mean transition rate is smaller than $\gamma$ and for fast driving $\lambda>\gamma$ it is larger than $\gamma$ [Fig. 5(a)]. In the Poisson case $\lambda=\gamma$, the mean transition rate equals exactly the rate of the dichotomous driving, i.e., $r=\lambda=\gamma$, as already stated.

Since $\left\langle\Delta T_{1}^{2}\right\rangle=\left\langle T_{1}^{2}\right\rangle-\left\langle T_{1}\right\rangle^{2}$, the CV is obtained as

$$
C_{V}=\sqrt{1+2 \frac{\left(k^{2}-\gamma^{2}\right)\left(\gamma^{2}-\lambda^{2}\right)}{(k+\lambda)^{2}\left(\gamma^{2}+2 k \lambda\right)}} .
$$

This expression predicts that the CV can be larger than, equal to, or smaller than 1 , depending on the switching rate $\lambda$ [Fig. 5(b)]. For slow driving, i.e., $\lambda<\gamma$, we find a CV larger than 1 . This can be intuitively understood if we consider the limit $\lambda \rightarrow 0$ of a vanishing switching rate. In this case, the sequence of RT's can be decomposed into two subgroups of RT's depending on whether the RT's belong to the upper or 

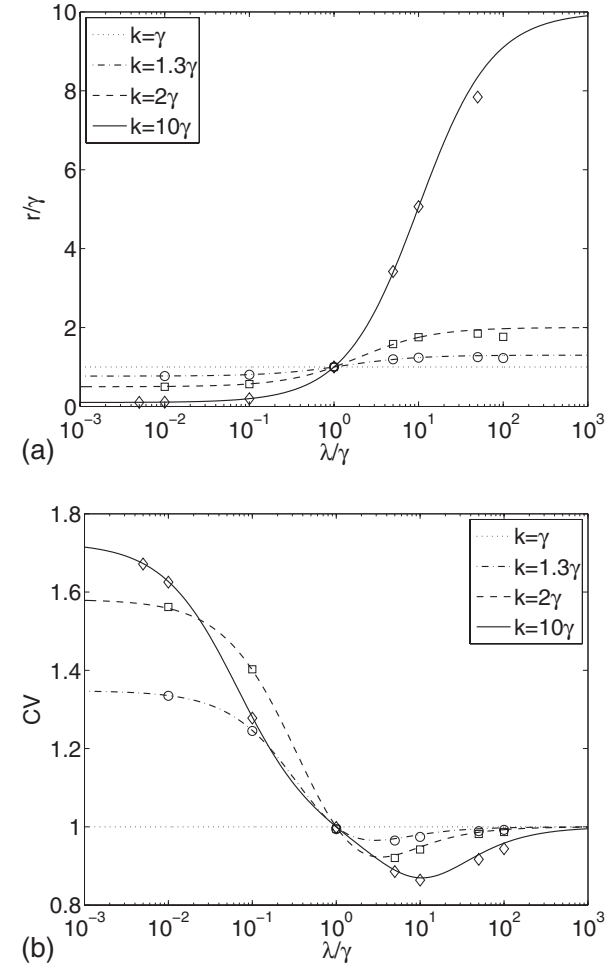

FIG. 5. (a) Mean transition rate (inverse mean RT) and (b) CV as a function of $\lambda / \gamma$ are depicted for different asymmetry parameters $k$ (as indicated). Lines correspond to the theoretical results Eqs. (24) and (26); symbols correspond to simulations of the Langevin equation (3) $(\bigcirc: k=1.3 \gamma, \square: k=2 \gamma, \diamond: k=10 \gamma)$. The parameters for the simulation were chosen as $D=0.05$ and, depending on $k, \sigma$ was computed according to the MFPT formula (7) (see Table I).

lower well. If we keep the sequential order in each subgroup, we obtain two Poisson processes with rate $k_{+}$and $k_{-}$, respectively. Thus, mean and standard deviations are equal to the values $m_{u}=1 / k_{+}$and $m_{l}=1 / k_{-}$, respectively. Now consider the mean $m$ and variance $v$ of the total RT sequence. The mean is given by $m=\left(m_{l}+m_{u}\right) / 2$, whereas the variance is equal to the mean variance $\left(m_{l}^{2}+m_{u}^{2}\right) / 2$ of the subgroups plus the variance of the means $\left(m_{l}^{2}+m_{u}^{2}\right) / 2-m^{2}$ by virtue of the law of total variance. Thus, we find $v=m_{l}^{2}+m_{u}^{2}-m^{2}>m^{2}$, which indeed implies $C_{V}=\sqrt{v} / m>1$.

Moreover, the Poisson case $\lambda=\gamma$ recovers the well-known result $C_{V}=1$ that holds for any (homogeneous) Poisson process. Furthermore, for $\lambda>\gamma$ the $\mathrm{CV}$ becomes smaller than 1 and it even attains a minimum at a certain optimal switching rate, where the jump process is most regular. However, if the switching becomes so fast that the particle virtually experiences the same average escape rate out of both metastable states, the $\mathrm{CV}$ approaches 1 again. It is easily verified that the $\mathrm{CV}$ as a function of both $k$ and $\lambda$ possesses a global minimum of $\sqrt{3} / 2 \approx 0.866$ and a global maximum of $\sqrt{3}$ $\approx 1.732$. Hence, the range of optimizing the $\mathrm{CV}$ is rather limited and the effect of a regularization of the output is rather weak.
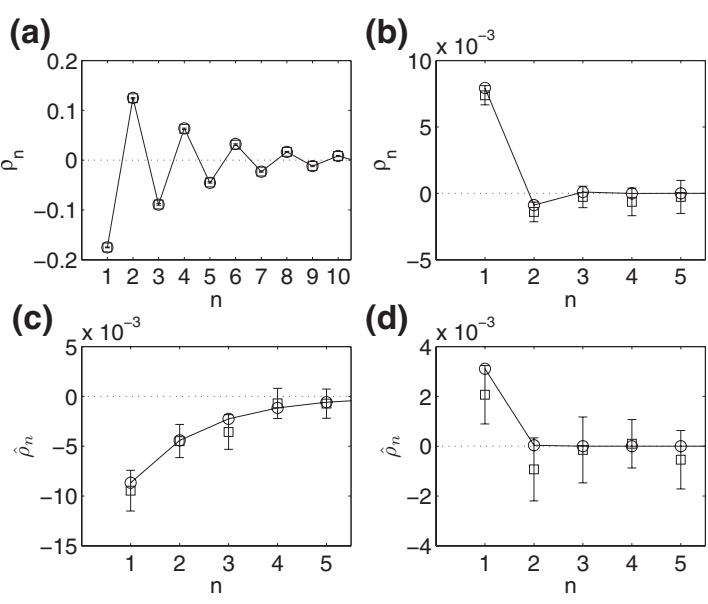

FIG. 6. SCC of RT's as a function of the lag for slow driving with $\lambda=0.1 \gamma$ (a) and fast driving with $\lambda=2 \gamma$ (b). In both cases $k$ $=2 \gamma$. Theoretical results [Eq. (28)] are represented by circles, the SCC's obtained from the simulation of the Langevin equation (3) are represented by squares. The lower panel shows the corresponding SCC of the SP. (c) For slow driving $\lambda=0.1 \gamma$ and (d) fast driving $\lambda=2 \gamma$. The parameters for the simulation were $D=0.05$, and $\sigma$ was computed corresponding to $k=2 \gamma$ by the MFPT formula (see Table I).

\section{Serial correlation coefficient}

For $n>1$, the system (23) can be written in the form of the recurrence relation

$$
\left(\begin{array}{l}
\bar{f}_{n} \\
\bar{P}_{n}
\end{array}\right)=g(s)\left(\begin{array}{cc}
2 k \lambda+\gamma^{2} & \gamma^{2} s \\
2(k+\lambda)+s & -\gamma^{2}
\end{array}\right)\left(\begin{array}{l}
\bar{f}_{n-1} \\
\bar{P}_{n-1}
\end{array}\right),
$$

which permits together with Eqs. (22) and (15) the calculation of the SCC. The result has been presented in [18], which states that $\rho_{n}$ is given by the alternating geometric sequence

$$
\rho_{n}=C\left[-\frac{\gamma^{2}}{2 k \lambda+\gamma^{2}}\right]^{n}
$$

with

$$
C=\frac{\left(k^{2}-\gamma^{2}\right)\left(\gamma^{2}-\lambda^{2}\right)}{2 k \lambda\left(k^{2}+\lambda^{2}+\gamma^{2}\right)+3 \gamma^{2}\left(k^{2}+\lambda^{2}\right)+2\left(k^{2} \lambda^{2}-\gamma^{4}\right)} .
$$

A proof of this formula is presented in the Appendix. This surprisingly simple expression clearly reveals an alternating behavior and an exponential decay of the SCC with respect to the lag $n$. The main features and the consistency with simulations have been discussed in [18]. For comparison with the behavior of the stochastic periods (see below), theory and simulation results for $\rho_{n}$ are depicted in Figs. 6(a) and 6(b).

For a slow switching rate $\lambda<\gamma$ [cf. Fig. 6(a)], the coefficient $C$ is positive, which implies that the SCC's $\rho_{1}, \rho_{3}, \rho_{5}, \ldots$ at odd lags are negative, whereas the SCC's $\rho_{2}, \rho_{4}, \rho_{6}, \ldots$ at even lags are positive. This is exactly the behavior that one would expect for a slow driving [18]: subsequent RT's consist of an (in the mean) longer RT (escape 
(a)

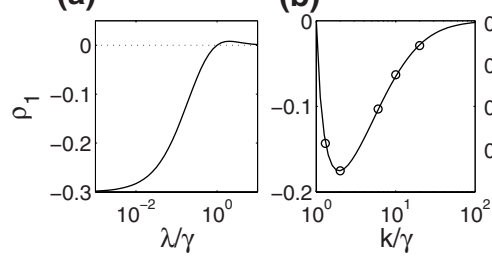

(c)

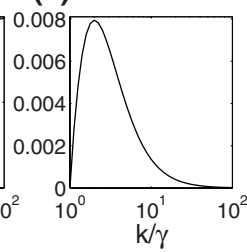

(d)

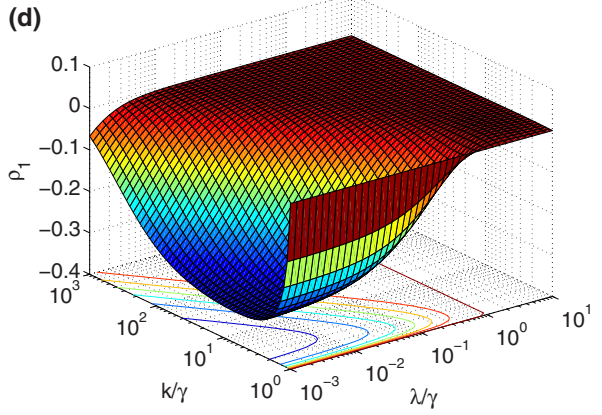

FIG. 7. (Color online) SCC of adjacent RT's $\rho_{1}$ is shown as a function of switching rate $\lambda$ and asymmetry $k$ : (a) $\rho_{1}$ as a function of $\lambda / \gamma$ for $k=2 \gamma$, (b) $\rho_{1}$ as a function of $k / \gamma$ for slow switching rate $\lambda=0.1 \gamma$, and (c) for fast switching rate $\lambda=2 \gamma$. Symbols in (b) represent simulation results for $\rho_{1}$. The lower panel (d) shows a 3D plot of $\rho_{1}$ as a function of both $\lambda$ and $k$.

over the larger barrier) and a shorter RT (escape over the smaller barrier) or the reversed succession-in any case, deviations of the two adjacent RT's go statistically in different directions and thus adjacent RT's are anticorrelated.

In the case of fast switching, i.e., $\lambda>\gamma[$ cf. Fig. 6(b)], the prefactor $C$ becomes negative, which leads to a sign inversion of the correlation coefficients. This entails an alternating behavior the opposite way around: the serial correlations $\rho_{1}, \rho_{3}, \rho_{5}, \ldots$ are now positive, while $\rho_{2}, \rho_{4}, \rho_{6}, \ldots$ are negative. As expected, all correlations between intervals vanish in the Poisson case $\lambda=\gamma$.

Knowing the SCC of the RT's, we can also calculate the SCC of the stochastic periods via Eq. (17). The result can be expressed by the SCC of the RT at odd lags,

$$
\hat{\rho}_{n}=\frac{2 k^{2} \lambda^{2}}{\left(1+\rho_{1}\right)\left(2 k \lambda+\gamma^{2}\right)^{2}} \rho_{2 n-1} .
$$

Interestingly, this correlation coefficient vanishes for $\lambda$ $\rightarrow 0$ as $\lambda^{2}$ [note that the factor $\left(1+\rho_{1}\right)>0$ ], i.e., for a very slow driving the stochastic periods are not correlated anymore, in marked contrast to the residence times. At finite $\lambda$ $>0$, the correlations of the stochastic periods have the same sign as $\rho_{2 n-1}$ due to the positive prefactor. This also includes the inversion of the correlations' sign at $\lambda=\gamma$. The SCC of the stochastic periods exhibits a purely exponential decay that is either negative [slow driving, Fig. 6(c)] or positive [fast driving, Fig. 6(d)]. In general, there is a good agreement with results of numerical simulations if for the rates the firstpassage time Eq. (7) is used.

Our analytical results for the SCC at lag one are plotted vs $\lambda$ and $k$ in Fig. 7. Two features become apparent: (i) the correlations are clearly maximized for slow driving; (ii) for
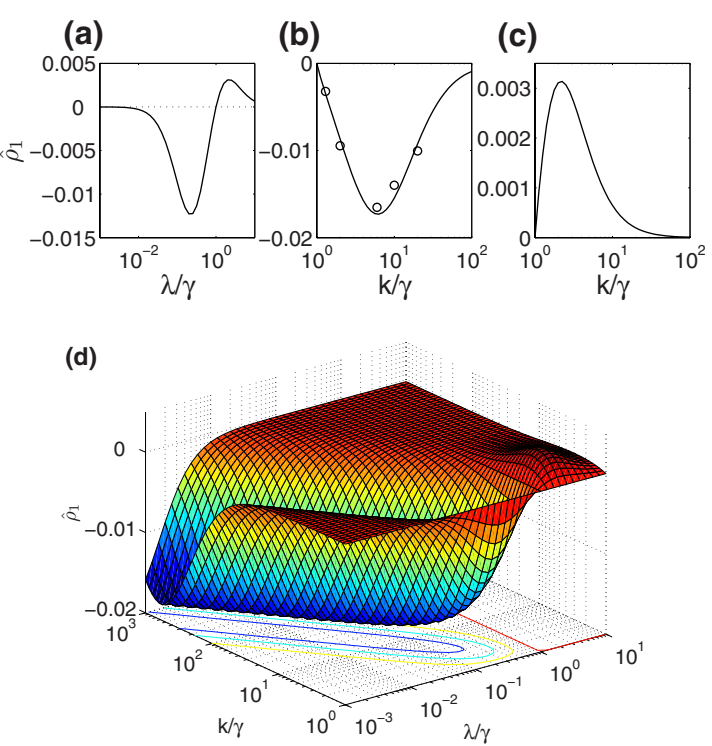

FIG. 8. (Color online) SCC of adjacent SP's $\hat{\rho}_{1}$ is shown as a function of switching rate $\lambda$ and asymmetry $k$ : (a) $\hat{\rho}_{1}$ as a function of $\lambda / \gamma$ for $k=2 \gamma$, (b) $\hat{\rho}_{1}$ as a function of $k / \gamma$ for slow switching rate $\lambda=0.1 \gamma$, and (c) for fast switching rate $\lambda=2 \gamma$. Symbols in (b) represent simulation results for $\hat{\rho}_{1}$. The lower panel (d) shows a $3 \mathrm{D}$ plot of $\hat{\rho}_{1}$ as a function of both $\lambda$ and $k$.

any fixed value $\lambda$ there exists an optimal value of $k / \gamma$, i.e., an optimal amplitude of the dichotomous forcing that maximizes the SCC of the residence time. The second feature is also verified by numerical simulation results [cf. Fig. 7(b)] can be explained as follows. On the one hand, it is plausible that the forcing can get too strong to see pronounced correlations: for very strong forcing, the output just follows the input dichotomous signal, which does not show any interval correlations by assumption. On the other hand, for vanishing driving amplitude, the response will not be correlated either. Thus, the correlation is maximized at finite $k$. The saturation seen for $\rho_{1}$ (RT) at vanishing $\lambda$ corresponds simply to the value of negative correlation between the RT's in the two potential minima in a potential with static bias.

Turning to the SCC of the stochastic periods (Fig. 8), we find similar maxima vs $k$ that, however, depend on the value of $\lambda$. The shape of the surface at slow driving $(\lambda \rightarrow 0)$ suggests a dependence on a single argument

$$
a=k \lambda / \gamma^{2}
$$

which yields after insertion into Eq. (30) and neglecting all remaining powers in $\lambda$

$$
\hat{\rho}_{1} \approx-\frac{2 a^{4}}{\left(4 a^{4}+8 a^{3}+2 a^{2}\right)(2 a+1)^{2}} .
$$

This function has a local minimum at

$$
a_{\min }=\frac{\sqrt{10} \cos [\arctan (3 \sqrt{111}) / 3]-1}{3} \approx 0.585
$$




$$
\hat{\rho}_{1}\left(a_{\min }\right) \approx-0.018 \text {. }
$$

The latter value is apparently the maximal (negative) correlation of the stochastic periods that we can achieve in the bistable system. The scaling $k_{\min } \lambda_{\min } / \gamma^{2} \approx 0.585$ for the optimal SP correlations suggests that the latter are of dynamic origin-for a static tilting of the bistable potential, the stochastic periods will be uncorrelated.

From the discussion above, it has become clear that RT correlations are most significant in the case of slow dichotomous driving. In this case, the measurement of correlations within a RT sequence could be used to estimate the switching rate $\lambda[18]$. Here, we derive an explicit expression for the switching rate in the case $\lambda \ll \gamma$, i.e., in the case of longcorrelated noise. Then from the correlation coefficients $\rho_{1}$ and $\rho_{2}$ one could calculate the ratio $q=\rho_{2} / \rho_{1}=-\gamma^{2} /\left(\gamma^{2}\right.$ $+2 k \lambda)<0$. Another measurable quantity is the mean RT or its inverse the mean transition rate $r=\left(k \lambda+\gamma^{2}\right) /(k+\lambda) \approx \lambda$ $+\gamma^{2} / k$. If we eliminate $\gamma^{2}$, we find the useful input-output relation

$$
\lambda=\frac{1+q}{1-q} r \quad(q<0),
$$

which holds for slow driving and arbitrary asymmetry. Hence, the (possibly) unknown parameter $\lambda$ of the driving can be expressed by the use of higher-order statistics.

\section{Fano factor and effective diffusion coefficient}

For a stationary counting process $N(t)$, the mean and variance are given by [28]

$$
\langle N(t)\rangle=r t
$$

and

$$
\operatorname{var}\{N(t)\}=r t+2 r \int_{0}^{t}\left(t-t^{\prime}\right) h\left(t^{\prime}\right) d t^{\prime}-r^{2} t^{2}
$$

where $r=\left(k \lambda+\gamma^{2}\right) /(\lambda+k)$ is the inverse mean RT and $h(t)$ is the conditional probability density that there is a transition at $t$ provided that there was a transition at $t=0$. In our case, this quantity can be written as

$$
h(t)=\sum_{n=1}^{\infty} f_{n}(t) .
$$

Summing up the first equation of Eq. (20) and noting that $\sum_{n=1}^{\infty} P_{n}(t)=1$, we find

$$
\dot{h}=-2(\lambda+k) h+2\left(k \lambda+\gamma^{2}\right) \text {. }
$$

This can be solved using the initial condition $h(0)=f_{1}(0)$ $=\gamma^{2}(k+\lambda) /\left(k \lambda+\gamma^{2}\right)$ resulting in

$$
h(t)=r+\left(\frac{\gamma^{2}}{r}-r\right) e^{-2(\lambda+k) t} .
$$

Using Eq. (36), we obtain

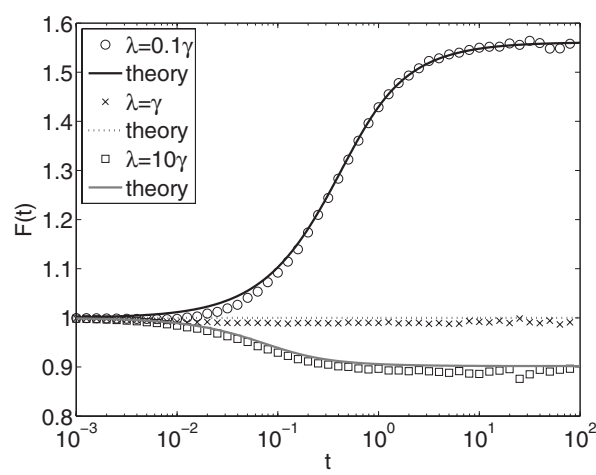

FIG. 9. The Fano factor as a function of the time window length $t$ for different switching rates of the dichotomous driving. The asymmetry of the bistable process was chosen as $k=2 \gamma$. Symbols represent the results obtained from simulations of the Langevin equation (3). The parameters used for the simulations were $D$ $=0.05$ and $\sigma$ corresponds to $k=2 \gamma$ as given in Table I.

$$
\operatorname{var}\{N(t)\}=\left(r+\frac{\gamma^{2}-r^{2}}{k+\lambda}\right) t+\frac{r^{2}-\gamma^{2}}{2(k+\lambda)^{2}}\left(1-e^{-2(k+\lambda) t}\right)
$$

and, hence, the Fano factor is

$$
F(t)=1+\frac{\left(k^{2}-\gamma^{2}\right)\left(\gamma^{2}-\lambda^{2}\right)}{(k+\lambda)^{2}\left(k \lambda+\gamma^{2}\right)} \times\left[1-\frac{1-e^{-2(k+\lambda) t}}{2(k+\lambda) t}\right] .
$$

From this expression, one can see that the Fano factor is 1 for $t \rightarrow 0$ and monotonically increases (decreases) for $\lambda<\gamma$ $(\lambda>\gamma)$ when the length of the time window $t$ is increased (Fig. 9). In the Poisson case $\lambda=\gamma$, the Fano factor is identically 1, as expected. For infinitely large time windows, i.e., $t \rightarrow \infty$, the Fano factor saturates at

$$
F_{\infty}=1+\frac{\left(k^{2}-\gamma^{2}\right)\left(\gamma^{2}-\lambda^{2}\right)}{(k+\lambda)^{2}\left(k \lambda+\gamma^{2}\right)} .
$$

In the asymptotic limit $t \rightarrow \infty$, the variance grows linearly in time. Thus, from Eq. (37) we find the effective diffusion coefficient

$$
D_{\mathrm{eff}}=\frac{\left(k \lambda+\gamma^{2}\right)\left[\lambda^{2}+k(k+\lambda)-\gamma^{2}\right]}{2(k+\lambda)^{3}} .
$$

For a given asymmetry $k$ in the range $\gamma \leqslant k<\sqrt{3} \gamma$ (weak driving), the effective diffusion coefficient possesses a minimum at the optimal switching rate

$$
\lambda_{\text {opt }}=\sqrt{\frac{1}{2}\left(3 \gamma^{2}-k^{2}\right)} \leqslant \gamma
$$

(Fig. 10). This means that at this switching rate of the input signal, the "phase" of the output is most coherent. At $k$ $=\sqrt{3} \gamma$ the minimum disappears and the effective diffusion coefficient is a monotonically increasing function of $\lambda$ for $k \geqslant \sqrt{3} \gamma$. In the limit of large asymmetry, we find 


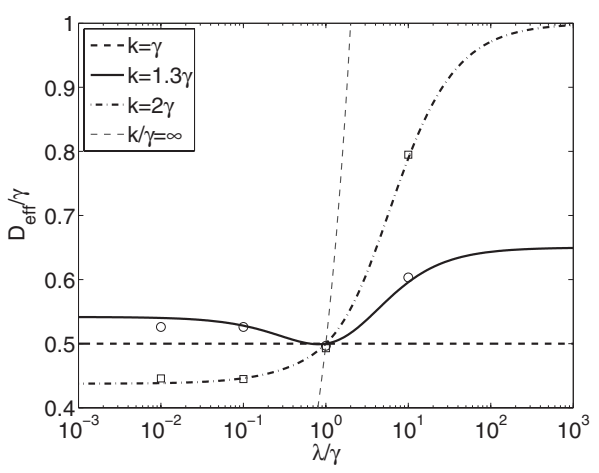

FIG. 10. Effective diffusion coefficient according to Eq. (39) as a function of the switching rate of the dichotomous driving for different asymmetry parameters $k$ (as indicated). The case $k / \gamma$ $\rightarrow \infty$ is indicated by the graph $D_{\text {eff }}=\lambda / 2$ [Eq. (41)]. Symbols represent the results of simulations carried out for the Langevin equation (3) with $D=0.05$ and the driving amplitudes $\sigma$ that correspond to $k=1.3 \gamma$ (circles) and $k=2 \gamma$ (squares); see Table I.

$$
\lim _{k \rightarrow \infty} D_{\text {eff }}=\frac{\lambda}{2}
$$

As a consequence, the effective diffusion coefficient can be arbitrarily small if only the driving is sufficiently slow and strong.

\section{E. Binary spectrum}

To investigate the spectral properties of the bistable process $x(t)$, we consider the power spectrum of the two-state filtered process $\tilde{x}(t)$, which takes the value $\tilde{x}=-1$ if the system is in the state $X_{-}$and $\tilde{x}=1$ if the system resides in the state $X_{+}$. The spectrum of this binary process will be called the binary spectrum. To calculate the binary spectrum, we consider the autocorrelation function $C(t)=\langle\widetilde{x}(t) \widetilde{x}(0)\rangle$. Using the two-point joint probabilities of $\widetilde{x}(t)$, we can write

$$
\begin{aligned}
\langle\widetilde{x}(t) \widetilde{x}(0)\rangle= & P(1, t ; 1,0)+P(-1, t ;-1,0)-P(1, t ;-1,0) \\
& -P(-1, t ; 1,0)=2[P(-1, t \mid-1,0)-P(1, t \mid \\
& -1,0)] P(-1,0)=P(-1, t \mid-1,0)-P(1, t \mid-1,0) .
\end{aligned}
$$

The conditional probabilities to find the particle on the left or right side at time $t$ are

$$
P(-1, t \mid-1,0)=\sum_{i=1}^{\infty} P_{2 i-1}(t)
$$

and

$$
P(+1, t \mid-1,0)=\sum_{i=1}^{\infty} P_{2 i}(t),
$$

respectively. Differentiating and using Eq. (20), we obtain

$$
\dot{C}(t)=2 \sum_{i=1}^{\infty}(-1)^{i} f_{i}(t) \text {. }
$$

Differentiating two more times and using repeatedly Eqs. (20) and (42), we find the third-order differential equation

$$
\dddot{C}=-2(k+\lambda) \ddot{C}-4 k \lambda \dot{C} .
$$

One obvious initial condition for this equation yields $C(0)$ $=1$. The other initial conditions can be found as follows: the probability that $\eta(0)= \pm \sigma$ given that at time $t=0$ the system resides in the state $X_{-}$is

$$
p\left( \pm \sigma \mid X_{-}\right)=\frac{p_{s}\left(X_{-}, \pm \sigma\right)}{p_{s}\left(X_{-}\right)}=2 p_{s}\left(X_{-}, \pm \sigma\right) .
$$

Using Eq. (5), we find $p\left( \pm \sigma \mid X_{-}\right)=\left(k_{\mp}+\lambda\right) /[2(k+\lambda)]$. Hence, the probability density for a transition at time $t=0$ is given by

$$
f_{1}(0)=k_{+} p\left(\sigma \mid X_{-}\right)+k_{-} p\left(-\sigma \mid X_{-}\right)=\frac{k \lambda+\gamma^{2}}{k+\lambda}
$$

and $f_{n}(0)=0$ for $n>1$. Note that this initial value for $f_{1}(t)$ is different compared to the previous sections [cf. Eq. (11)], where we required that the origin $t=0$ coincides with a transition time. Using Eq. (42), we finally obtain $\dot{C}(0)=-2(k \lambda$ $\left.+\gamma^{2}\right) /(k+\lambda)$ and $\ddot{C}(0)=4 \gamma^{2}$. The solution of Eq. (43) subject to these initial conditions yields the autocorrelation function for $t \geqslant 0$,

$$
C(t)=\frac{\left(\gamma^{2}-\lambda^{2}\right) e^{-2 k t}+\left(k^{2}-\gamma^{2}\right) e^{-2 \lambda t}}{k^{2}-\lambda^{2}} .
$$

We can now apply the Wiener-Khinchin theorem to obtain the spectrum $S(\omega)$,

$$
\begin{aligned}
S(\omega) & =2 \int_{0}^{\infty} C(t) \cos \omega t d t \\
& =\frac{16 k \lambda\left(k^{2}+k \lambda+\lambda^{2}-\gamma^{2}\right)+4\left(k \lambda+\gamma^{2}\right) \omega^{2}}{(k+\lambda)\left(4 k^{2}+\omega^{2}\right)\left(4 \lambda^{2}+\omega^{2}\right)} .
\end{aligned}
$$

Figure 11 shows that this expression for $S(\omega)$ is in excellent agreement with the spectrum of the filtered process $\widetilde{x}(t)$ obtained from numerical simulations of Eq. (3).

The shape of the binary spectrum turns out to be particularly useful in the slow driving case $\lambda \ll \gamma$. In this case, we find that $S(\omega)$ consists of two well-separated Lorentzian functions. Indeed, for high frequencies the spectrum behaves asymptotically like

$$
S_{\text {high }}(\omega)=\frac{4\left(k \lambda+\gamma^{2}\right)}{k} \frac{1}{(2 k)^{2}+\omega^{2}},
$$

whereas at low frequencies the spectrum can be written as

$$
S_{\text {low }}(\omega)=\frac{4\left(k^{2}-\gamma^{2}\right)}{k^{2}} \frac{\lambda}{(2 \lambda)^{2}+\omega^{2}} .
$$

This is illustrated in Fig. 12 and reveals that the spectrum provides a simple estimation of the driving parameters $\lambda$ and $k$, since the half-width at half maximum is $2 \lambda$ and $2 k$, respectively. Thus, the binary spectrum offers an alternative way to extract parameters of the dichotomous noise.

\section{CONCLUSION}

The master equation approach developed by the authors in [18] permitted a theoretical analysis of the RT sequence of a 

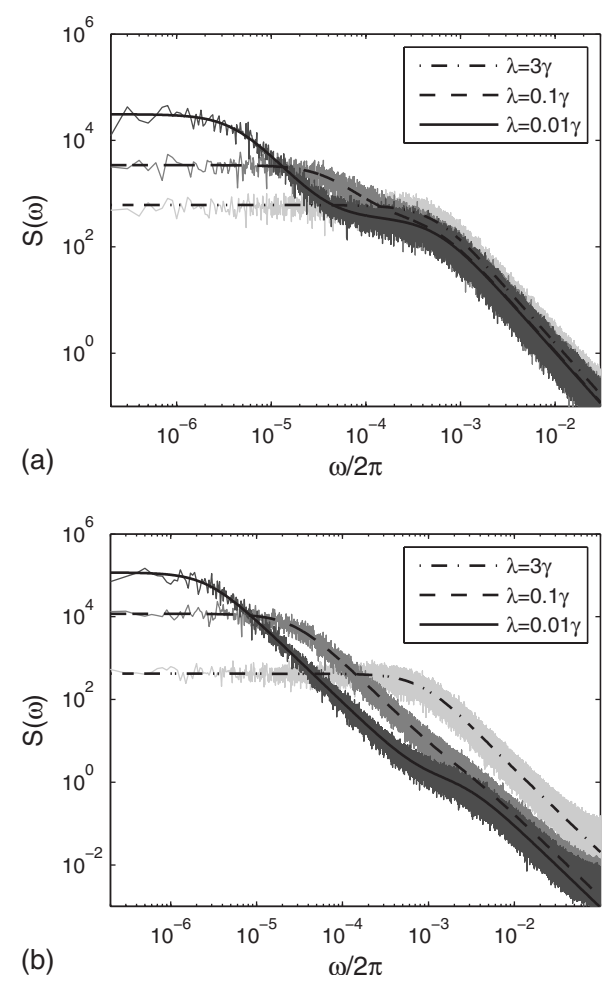

FIG. 11. Spectrum of the two-state filtered process as a function of the frequency $f=\omega / 2 \pi$ for different switching rates $\lambda$ (as indicated in the legend). The spectra are depicted for weak forcing with $\sigma=0.04$ (a) and for strong forcing with $\sigma=0.1585$ (b). There is a Lorentzian decay for high frequencies and a separated second decay in the spectrum in the case of slow switching. Here, $D=0.05$ and the value for $\gamma$ and $k$ can be seen from Table I; thick lines display the theory and thin lines display the corresponding simulated spectra.

bistable system that is driven by dichotomous colored noise. Based on this technique, we derived several statistical measures as a function of the driving parameters $\lambda$ and $k$, including the coefficient of variation (first-order RT statistics), the serial correlation coefficient of the stochastic periods (higher-order RT statistics), the Fano factor, and the effective diffusion coefficient (count statistics), as well as the binary

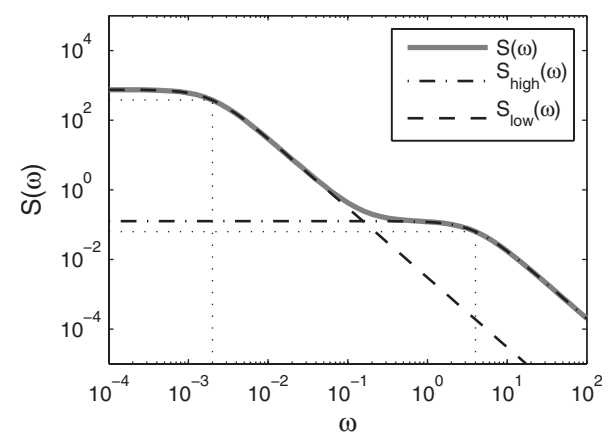

FIG. 12. Illustration of the binary spectrum of a slowly driven system with driving parameters $\lambda=0.001 \gamma, k=2 \gamma$, and $\gamma=2$ according to Eqs. (45)-(47). It exhibits two well-separated Lorentzian decays with half-width at half maximum of $\omega=2 \lambda$ and $\omega=2 k$ as indicated by the dashed lines. spectrum of the bistable process. We also proved the formula for the SCC of the RT's, which was discussed in detail in [18]. The results are exact within the rate description, i.e., the only assumption made is that the escape rates of the metastable states are approximately constant.

We found that an external dichotomous driving with a switching rate that exactly matches the geometric mean of the transition rates $\gamma$ makes the output sequence Poissonian. In Kramers' case of weak driving, this geometric mean rate $\gamma$ coincides with the escape rate of the symmetric, undriven system. The Poissonian nature at $\lambda=\gamma$ explains why all correlations vanish at that switching rate and why the $\mathrm{CV}$ and the Fano factor are in this case unity regardless of the system's asymmetry $k$. The geometric mean rate $\gamma$, therefore, establishes a characteristic (inverse) time scale of the system. If the switching rate is not equal to $\gamma$, the output statistics exhibits qualitatively different properties depending on whether $\lambda<\gamma$ (slow driving) or $\lambda>\gamma$ (fast driving). This becomes particularly obvious for the SCC, which changes its sign at all lags for the characteristic switching rate $\lambda=\gamma$. In general, the slow driving regime is richer in interesting features such as (i) large variability $\left[C_{V}>1, F(t)>1\right]$; (ii) strong interval correlations (that allow for an estimation of $\lambda$ [18]); (iii) minimal phase diffusion; and (iv) two Lorentzian decays in the binary spectrum, which permit an estimation of the driving parameters.

A problem where our method may be also applied is the case of a periodic driving force rather than a stochastic driving. Note that a periodic forcing is an example for input processes with an infinitely large correlation time. This case yields the standard model of stochastic resonance, which has been the subject of numerous studies (see [14] and references therein). However, higher-order RT statistics and its possible role for stochastic resonance have not been investigated so far. With the techniques employed in this paper, the calculation of the SCC in a periodically driven system seems to be feasible, at least in the case of a weak driving. The question to what extend the RT correlations characterize other aspects of the stochastic resonance phenomenon remains an interesting subject for future studies.

\section{ACKNOWLEDGMENTS}

We would like to thank Lutz Schimansky-Geier for helpful discussions and his support of this project. T.S. thanks $H$. Nakahara and German National Academic Foundation for support.

\section{APPENDIX: PROOF OF EQ. (28)}

Here, a proof of the formulas (28) and (29) is given. In the first step, the correlation coefficients $\rho_{1}$ and $\rho_{2}$ can be explicitly calculated using Eq. (15), (22), and (23), which confirms that

$$
\rho_{1}=C q, \quad \rho_{2}=C q^{2},
$$

where $q=-\frac{\gamma^{2}}{2 k \lambda+\gamma^{2}}$ is the common ratio of the sequence (28).

In the second step, we show that for $n>2$, the assumption $\rho_{n-1}=q \rho_{n-2}$ implies $\rho_{n}=q \rho_{n-1}$. This together with Eq. (A1) 
leads by mathematical induction to the assertion (28). At first, however, we introduce some properties that will be useful for further analysis. From Eq. (21), it follows that

$$
\bar{f}_{1}(0)=1
$$

and

$$
\bar{P}_{1}(0)=\frac{k+\lambda}{k \lambda+\gamma^{2}}=\left\langle T_{1}\right\rangle .
$$

Furthermore, we denote the matrix of the linear mapping Eq. (27) by

$$
\mathbf{A}(s)=g(s)\left(\begin{array}{cc}
2 k \lambda+\gamma^{2} & \gamma^{2} s \\
2(k+\lambda)+s & -\gamma^{2}
\end{array}\right),
$$

which for $s=0$ reduces to

$$
\mathbf{A}(0)=\left(\begin{array}{cc}
1 & 0 \\
2 \frac{k+\lambda}{2 k \lambda+\gamma^{2}} & -\frac{\gamma^{2}}{2 k \lambda+\gamma^{2}}
\end{array}\right)
$$

Using Eq. (A2), we find

$$
\bar{f}_{n}(0)=1
$$

for all $n \geqslant 1$, which simply expresses the normalization condition for $f_{n}(t)$. From Eq. (A3), it is easily found that $\bar{P}_{2}(0)$ $=\frac{k+\lambda}{k \lambda+\gamma^{2}}=\left\langle T_{1}\right\rangle=\bar{P}_{1}(0)$ and by induction we can state for all $n$
$\geqslant 1$

$$
\bar{P}_{n}(0)=\left\langle T_{1}\right\rangle .
$$

A final obvious property concerns the mean of the $n$ th-order interval, which for all $n \geqslant 1$ is given by

$$
\left\langle T_{n}\right\rangle=n\left\langle T_{1}\right\rangle .
$$

Because of Eq. (15), we consider the expression

$$
\begin{gathered}
\left\langle\Delta T_{n+1}^{2}\right\rangle-2\left\langle\Delta T_{n}^{2}\right\rangle+\left\langle\Delta T_{n-1}^{2}\right\rangle=\left\langle T_{n+1}^{2}\right\rangle-2\left\langle T_{n}^{2}\right\rangle+\left\langle T_{n-1}^{2}\right\rangle \\
-2\left\langle T_{1}\right\rangle^{2} .
\end{gathered}
$$

The second moment of $T_{n}$ can be reduced in the following way:

$$
\begin{aligned}
\left\langle T_{n}^{2}\right\rangle=\left.\frac{d^{2} \bar{f}_{n}}{d s^{2}}\right|_{s=0}= & {\left[\frac{d^{2}}{d s^{2}}\left(A_{11} \bar{f}_{n-1}+A_{12} \bar{P}_{n-1}\right)\right]_{s=0} } \\
= & {\left[A_{11}^{\prime \prime} \bar{f}_{n-1}+2 A_{11}^{\prime} \bar{f}_{n-1}^{\prime}+A_{11}(0) \bar{f}_{n-1}^{\prime \prime}\right.} \\
& \left.+A_{12}^{\prime \prime} \bar{P}_{n-1}+2 A_{12}^{\prime} \bar{P}_{n-1}^{\prime}\right]_{s=0} \\
= & A_{11}^{\prime \prime}(0)-2 A_{11}^{\prime}(0)(n-1)\left\langle T_{1}\right\rangle+\left\langle T_{n-1}^{2}\right\rangle \\
& +A_{12}^{\prime \prime}(0)\left\langle T_{1}\right\rangle+2 A_{12}^{\prime}(0) \bar{P}_{n-1}^{\prime}(0) .
\end{aligned}
$$

Here, we have again used Eqs. (A5)-(A8). All terms that are independent of $n$ or linear in $n$ will cancel out when inserted in the first three terms on the r.h.s. of Eq. (A9). Thus, we obtain

$$
\begin{aligned}
\left\langle\Delta T_{n+1}^{2}\right\rangle-2\left\langle\Delta T_{n}^{2}\right\rangle+\left\langle\Delta T_{n-1}^{2}\right\rangle= & \left\langle T_{n}^{2}\right\rangle-2\left\langle T_{n-1}^{2}\right\rangle+\left\langle T_{n-2}^{2}\right\rangle \\
& -2\left\langle T_{1}\right\rangle^{2}+2 A_{12}^{\prime}(0)\left[\bar{P}_{n}^{\prime}(0)\right. \\
& \left.-2 \bar{P}_{n-1}^{\prime}(0)+\bar{P}_{n-2}^{\prime}(0)\right] .
\end{aligned}
$$

It can be shown in the same way that the expression in brackets can be reduced as follows:

$$
\begin{aligned}
\bar{P}_{n}^{\prime}(0)-2 \bar{P}_{n-1}^{\prime}(0)+\bar{P}_{n-2}^{\prime}(0)= & A_{22}(0)\left[\bar{P}_{n-1}^{\prime}(0)-2 \bar{P}_{n-2}^{\prime}(0)\right. \\
& \left.+\bar{P}_{n-3}^{\prime}(0)\right] .
\end{aligned}
$$

Using the last two relations and taking into account that $A_{12}^{\prime}(0)=-A_{22}(0)=-q$, we find

$$
\begin{aligned}
2\left\langle\Delta T_{1}^{2}\right\rangle \rho_{n}= & \left\langle T_{n}^{2}\right\rangle-2\left\langle T_{n-1}^{2}\right\rangle+\left\langle T_{n-2}^{2}\right\rangle-2\left\langle T_{1}\right\rangle^{2}-2 q^{2}\left[\bar{P}_{n-1}^{\prime}(0)\right. \\
& \left.-2 \bar{P}_{n-2}^{\prime}(0)+\bar{P}_{n-3}^{\prime}(0)\right] .
\end{aligned}
$$

Subtracting from this relation the expression $2\left\langle\Delta T_{1}^{2}\right\rangle \rho_{n-1}$ $=\left\langle T_{n}^{2}\right\rangle-2\left\langle T_{n-1}^{2}\right\rangle+\left\langle T_{n-2}^{2}\right\rangle-2\left\langle T_{1}\right\rangle^{2}$, we obtain

$$
2\left\langle\Delta T_{1}^{2}\right\rangle\left(\rho_{n}-\rho_{n-1}\right)=-2 q^{2}\left[\bar{P}_{n-1}^{\prime}(0)-2 \bar{P}_{n-2}^{\prime}(0)+\bar{P}_{n-3}^{\prime}(0)\right] .
$$

In an analogous manner, it is true that

$$
2\left\langle\Delta T_{1}^{2}\right\rangle\left(\rho_{n-1}-\rho_{n-2}\right)=-2 q\left[\bar{P}_{n-1}^{\prime}(0)-2 \bar{P}_{n-2}^{\prime}(0)+\bar{P}_{n-3}^{\prime}(0)\right]
$$

and dividing both expressions yields

$$
\rho_{n}-\rho_{n-1}=q\left(\rho_{n-1}-\rho_{n-2}\right) .
$$

According to the assumption, it is $q \rho_{n-2}=\rho_{n-1}$ and hence $\rho_{n}$ $=q \rho_{n-1}$.
[1] H. Risken, The Fokker-Planck Equation (Springer, Berlin, 1984).

[2] R. Ratnam and M. E. Nelson, J. Neurosci. 20, 6672 (2000).

[3] M. J. Chacron, A. Longtin, and L. Maler, J. Neurosci. 21, 5328 (2001).

[4] S. B. Lowen and M. C. Teich, J. Acoust. Soc. Am. 92, 803 (1992).

[5] M. J. Chacron, K. Pakdaman, and A. Longtin, Neural Comput. 15, 253 (2003).

[6] J. W. Middleton, M. J. Chacron, B. Lindner, and A. Longtin,
Phys. Rev. E 68, 021920 (2003).

[7] B. Lindner, Phys. Rev. E 69, 022901 (2004).

[8] M. J. Chacron, B. Lindner, and A. Longtin, Phys. Rev. Lett. 92, 080601 (2004).

[9] B. Lindner, M. J. Chacron, and A. Longtin, Phys. Rev. E 72, 021911 (2005).

[10] N. Lüdtke and M. E. Nelson, Neural Comput. 18, 2879 (2006).

[11] H. A. Kramers, Physica (Amsterdam) 7, 284 (1940).

[12] P. Hänggi, P. Talkner, and M. Borkovec, Rev. Mod. Phys. 62, 
251 (1990).

[13] B. McNamara and K. Wiesenfeld, Phys. Rev. A 39, 4854 (1989).

[14] L. Gammaitoni, P. Hänggi, P. Jung, and F. Marchesoni, Rev. Mod. Phys. 70, 223 (1998).

[15] P. Pechukas and P. Hänggi, Phys. Rev. Lett. 73, 2772 (1994).

[16] L. S. Tsimring and A. Pikovsky, Phys. Rev. Lett. 87, 250602 (2001).

[17] C. Masoller, Phys. Rev. Lett. 90, 020601 (2003).

[18] B. Lindner and T. Schwalger, Phys. Rev. Lett. 98, 210603 (2007).

[19] P. Hänggi and P. Jung, Adv. Chem. Phys. 89, 239 (1995).

[20] A. Neiman, A. Silchenko, V. Anishchenko, and L. Schimansky-Geier, Phys. Rev. E 58, 7118 (1998).

[21] A. Neiman, L. Schimansky-Geier, F. Moss, B. Shulgin, and J. J. Collins, Phys. Rev. E 60, 284 (1999).
[22] R. Rozenfeld, J. A. Freund, A. Neiman, and L. SchimanskyGeier, Phys. Rev. E 64, 051107 (2001).

[23] J. F. Lindner and A. R. Bulsara, Phys. Rev. E 74, 020105(R) (2006).

[24] I. Broussell, I. L'Heureux, and E. Fortin, Phys. Lett. A 225, 85 (1997).

[25] R. Mannella, in Stochastic Processes in Physics, Chemistry, and Biology, edited by J. A. Freund and T. Pöschel, Lecture Notes in Physics, Vol. 557 (Springer, Berlin, 2000), p. 353.

[26] D. T. Gillespie, J. Comput. Phys. 2, 403 (1976).

[27] L. Pontryagin, A. Andronov, and A. Witt, Zh. Eksp. Teor. Fiz. 3, 172 (1933), reprinted in Noise in Nonlinear Dynamical Systems, edited by F. Moss and P. V. E. McClintock (Cambridge University Press, Cambridge, 1989), Vol. 1, p. 329.

[28] D. R. Cox and V. Isham, Point Processes (Chapman and Hall, London, 1980). 